\title{
Benedikt Stimmer
}

\section{,Zivilisierung' durch Sprache?}

\author{
Die Verbreitung des Deutschen im Rahmen der habsburgischen \\ Schulpolitik in Galizien 1772-1790
}

\begin{abstract}
Zusammenfassung: Mit Galizien fiel 1772 ein großes und bevölkerungsreiches Gebiet an die Habsburgermonarchie, das den staatlichen Beamten von Beginn an als ,innere Kolonie‘ erschien. Vor dem Hintergrund der Vorstellung einer imperialen Zivilisierungsmission muss auch die Sprachenpolitik betrachtet werden, die sich in der neuen Provinz schon unter Maria Theresia eng mit schulpolitischen Fragen verband und auf eine öffentliche Durchsetzung des als Kultursprache verstandenen Deutschen gegenüber dem Polnischen abzielte. Unter Joseph II. berührte die kulturmissionarisch wie utilitaristisch legitimierte Expansion der deutschen Sprache noch stärker als zuvor den Elementarschulbereich. Eine besondere Ausprägung erfuhr diese volksaufklärerisch motivierte Politik im Bereich des jüdischen Schulwesens, in dem sich die Verbreitung des Deutschen explizit mit dem Wunsch nach einer sittlichmoralischen ,Besserung' der galizischen Juden verband. Neben dem Widerstand der jüdischen Gemeinden verdeutlichte spätestens 1790 vor allem die Opposition des galizischen Adels, der im 19. Jahrhundert eine weitgehende Zurückdrängung des Deutschen zugunsten des Polnischen erreichte, das Scheitern der josephinischen Politik.
\end{abstract}

Schlagwörter: Galizien; Schulwesen; Sprachpolitik; deutsche Sprache; Zivilisierungsmission; Josephinismus; Habsburgermonarchie

Abstract: In 1772 the Habsburg Monarchy acquired the extensive and densely populated province of Galicia which, from the beginning, was regarded as an 'inland colony' by state officials. This understanding of a civilizing mission in the imperial East provided the discursive foundation for early Austrian language policy. It was one which generally favoured German over the vernacular Polish and was inherently linked to the establishment of Maria Theresa's new school system in the multi-ethnic region. Under Joseph II, the expansion of the German language appears to be even more strongly connected to the development of elementary schools in Galicia. This interrelation, motivated by the Emperor's desire for popular Enlightenment as well as the consolidation of power, manifested itself most forcefully within the public Jewish school system, where Germanisation was managed as a means of civilization towards Galicia's Jewish population. The failure of these language policies was evident at latest by 1790, when the Polish nobility openly resisted the dissemination of German in the province and were able to enforce a process of Polonization over the course of the 19th century.

Keywords: Galicia; school system; language policy; German language; civilizing mission; Josephinism; Habsburg Monarchy

Benedikt Stimmer, M. A., Aslangasse 2/4/6, 1190 Wien, Österreich, benedikt.stimmer@yahoo.de 


\section{Eine habsburgische mission civilisatrice}

Von ,Zivilisierungsmissionen“ ist traditionell meist im kolonialen Kontext die Rede, vor allem im Zusammenhang mit dem Imperialismus des 19. Jahrhunderts. Dass gerade die Teilungen Polens, bei denen große, in Westeuropa oft als rückständig geltende Gebiete Ostmitteleuropas unter österreichische, preußische und russische Herrschaft gerieten, bislang fast ausnahmslos von jenen historiographischen Diskursen ausgeklammert wurden, bietet Anlass zur Verwunderung. Immerhin handelte es sich bei der Integration der neuen Provinzen vor allem im Fall der Habsburgermonarchie und Preußens um Zivilisierungsmissionen par excellence, da sich deren Monarchen als aufgeklärte Herrscher mit zivilisatorischem Sendungsbewusstsein begriffen und die Teilungen zudem schon von den Zeitgenossen vielfach unter diesem Gesichtspunkt wahrgenommen wurden. So war insbesondere die erste Aufteilung der von weiten Kreisen der aufgeklärt-bürgerlichen Gesellschaft als anarchisch und reformbedürftig angesehenen Rzeczpospolita für Johann Gottfried Seume etwa „unstreitig zur Wohltat für die Menschheit“, ${ }^{1}$ und auch Voltaire lobte 1772 das Teilungsprojekt („ce grand projet“) als „noble“ und „utile“.2

Ausgehend von diesen Überlegungen wird im Folgenden die habsburgische Regierungspraxis in Galizien bis zum Tod Kaiser Josephs II. 1790 in den Blick genommen - in jenem Gebiet also, das für die kaiserliche Bürokratie unter Zuhilfenahme kolonialer Raumanalogien ein österreichisches ,Sibirien ' darstellte und in der imperialen Wahrnehmung von Beginn an als periphere, unterentwickelte Region konstituiert wurde. ${ }^{3}$ Indem die habsburgische mission civilisatrice in Galizien unter einem sprachpolitischen Gesichtspunkt betrachtet wird, soll im Speziellen danach gefragt werden, inwieweit sich die Verbreitung der deutschen Sprache im Rahmen des neuen staatlichen Schulwesens als Ausdruck einer ,aufgeklärt-absolutistischen' Kulturmission werten lässt. In Anlehnung an Almut Steinbach, die einen ähnlichen Ansatz bereits im Zusammenhang mit der

1 Johann Gottfried Seume: Über das Leben und den Karakter der Kaiserin von Russland Katharina II. In: Ders.: Werke. Hg. v. Jörg Drews. Bd. 2. Frankfurt/M. 1993, S. 229-327, hier S. 259. Seume, der an den Verhandlungen selbst beteiligt war, diente die Auffassung der Teilungen als Zivilisierungsmission nicht zuletzt auch zur Selbstlegitimation. Vgl. Hans-Jürgen Bömelburg, Andreas Gestrich, Helga Schnabel-Schüle: Die Teilungen Polen-Litauens als Zäsur einer europäischen Strukturgeschichte. Komparative Überlegungen jenseits der Nationalgeschichtsschreibung. In: Dies. (Hg.): Die Teilungen Polen-Litauens. Inklusions- und Exklusionsmechanismen - Traditionsbildung - Vergleichsebenen. Osnabrück 2013, S. 9-35, hier S. 20 f.

2 Voltaire to Catherine II, empress of Russia. Ferney, 29.05.1772. In: Ders.: Correspondence and Related Documents. Hg.v. Theodore Besterman. Bd. 38: July 1771-July 1772. Oxford 2013 (The Complete Works of Voltaire 122), S. 401-403, hier S. 402.

3 Vgl. Klemens Kaps: Kulturelle Trennlinien und wirtschaftliche Konkurrenz. Galizische Modernisierungsdiskurse zwischen Subalternität und Dominanz in der zweiten Hälfte des 19. Jahrhunderts. In: Alexander Kratochvil, Renata Makarska, Katharina Schwitin, Annette Werberger (Hg.): Kulturgrenzen in postimperialen Räumen. Bosnien und Westukraine als transkulturelle Regionen. Bielefeld 2013 (Edition Kulturwissenschaft 11), S. 33-60, hier S. 34. 
Verbreitung des Englischen im 19. Jahrhundert in Ceylon und den Protected Malay States verfolgt hat, geht es also auch hier um die Sichtbarmachung von Sprache ,als eine derjenigen Dimensionen von Kultur, die für die Zivilisierungsmission [...] instrumentalisiert wurden “4 - schließlich muss imperiale Sprachpolitik nach dem Motto ,cuius regio, eius lingua' immer als Ausdruck von Machtverhältnissen begriffen werden. ${ }^{5}$

Um die Hintergründe und Überlegungen zur Expansion des Deutschen im galizischen Schulwesen seit Maria Theresia nachzuzeichnen, wird im Folgenden auf Aktenmaterial der bis 1791 bestehenden Studienhofkommission aus dem Allgemeinen Verwaltungsarchiv in Wien zurückgegriffen. In den meisten Fällen handelt es sich dabei um Korrespondenzen zwischen dem Gubernium in Lemberg (L'viv, Lwów) und der Wiener Zentralbehörde. Da aus der Fragestellung nahezu zwangsläufig eine Methodik hervorgeht, die sich der Identifikation nicht unmittelbar einsichtiger Diskurse verschreibt - immerhin kann davon ausgegangen werden, dass Zivilisierungsdiskurse in den meisten Fällen als dialogisierender Hintergrund der jeweiligen Quellen präsent sind -, wird bei der Analyse auf Anregungen der Historischen sowie vor allem auch der Kritischen Diskursanalyse zurückgegriffen.

\section{Die ,Revindikation‘ Galiziens}

1772 fiel rund ein Drittel des polnischen Staatsgebiets an die drei Teilungsmächte, wobei die Habsburgermonarchie mit Galizien den bevölkerungsreichsten Anteil erhielt. ${ }^{6}$ Die Zahl der Einwohner, die sich hauptsächlich aus unierten Ruthenen, katholischen Polen sowie einer bedeutenden jüdischen Minderheit zusammensetzten, betrug Mitte der 1770er Jahre gut zweieinhalb Millionen und stieg bis 1790 bereits auf deutlich über drei Millionen an. ${ }^{7}$ Neben der „militärische[n] Festigung des Grenzgebietes“ war das wichtigste Ziel der neuen Administration die „Anhebung des Lebensstandards der Bevölkerung im aufklärerischen Geist und mit politischen Hinterabsichten“, namentlich einer „Bindung der Bevölkerung an das neue Herrscherhaus“. ${ }^{8}$ Dass dies kein einfaches Unterfangen war, bemerkte schon 1772 der erste Provinz-

\footnotetext{
4 Almut Steinbach: Sprachpolitik und Zivilisierungsmission im Britischen Empire. Die Verbreitung der englischen Sprache im 19. Jahrhundert in Ceylon und den Protected Malay States. In: Boris Barth (Hg.): Zivilisierungsmissionen. Imperiale Weltverbesserung seit dem 18. Jahrhundert. Konstanz 2005 (Historische Kulturwissenschaft 6), S. 149-168, hier S. 152.

5 Vgl. Florian Coulmas: Sprache und Staat. Studien zur Sprachplanung und Sprachpolitik. Berlin-New York 1985 (Sammlung Göschen 2501), S. $114 \mathrm{f}$.

6 Vgl. Michael G. Müller: Die Teilungen Polens 1772 - 1793 - 1795. München 1984, S. 39.

7 Vgl. Rudolf A. Mark: Galizien unter österreichischer Herrschaft. Verwaltung - Kirche - Bevölkerung. Marburg 1994 (Historische und landeskundliche Ostmitteleuropa-Studien 13), S. 53-63.

8 Hans-Christian Maner: Galizien. Eine Grenzregion im Kalkül der Donaumonarchie im 18. und 19. Jahrhundert. München 2007 (Veröffentlichungen des Instituts für deutsche Kultur und Geschichte Südosteuropas an der Ludwig-Maximilians-Universität München. Wissenschaftliche Reihe Geschichte und Zeitgeschichte 111), S. 35.
} 
gouverneur Graf von Pergen, für den sich der „sehr schlecht[e]“ Zustand des Landes „der Einführung einer guten Regierung auf allen Seiten entgegen[stellte]“. ${ }^{9}$ Sein Vorschlag einer graduellen administrativen Eingliederung, die auf lokale Gegebenheiten Rücksicht nehmen sollte, stieß bei Maria Theresia und mehr noch bei ihrem Sohn Joseph jedoch auf wenig Gegenliebe - schließlich erschien dem Kaiser das Land ,in the light of his own Josephine sense of imperial mission“. ${ }^{10}$ Pergens rasche Absetzung änderte allerdings wenig an der langsamen Angleichung der galizischen Verhältnisse an jene in der restlichen Monarchie, lag diese doch primär darin begründet, dass die neue Provinz „keine Tradition als einheitliche Geschichtsregion besaß“, also eine künstlich geschaffene politische Einheit war. ${ }^{11}$

An dieser Künstlichkeit vermochte auch der habsburgische Legitimismus nichts $\mathrm{zu}$ ändern, der sich durch die historisch nicht begründete Grenzziehung sowie die Zuweisung der galizischen Angelegenheiten an die Böhmisch-Österreichische Hofkanzlei ${ }^{12}$ und trotz der Ansprüche, die von der ungarischen Königstitulatur abgeleitet wurden, selbst unterminierte. Vor allem das Verhältnis zum polnischen Adel musste sich in dieser Hinsicht konfliktträchtig gestalten, gründete sich die legitimistische Haltung Wiens doch auf das ,ius postliminii', das ,Rückkehrerrecht', das man für älter als die Privilegien der im Gesellschafts- und Wirtschaftsleben dominierenden Szlachta erachtete. ${ }^{13}$ Laut Hans-Jürgen Bömelburg stellte sich dieser Elitenkonflikt zwischen dem „barock erzogenen, von republikanischen und widerständigen Traditionen des Sarmatismus durchtränkten“ Adel und einer landfremden Beamtenschaft, „die nüchterne Staatsräson, Gehorsam und zweckmäßig-utilitaristisches Denken verinnerlicht hatte“, ${ }^{14}$ einer erfolgreichen Integration Galiziens in den Gesamtstaat entgegen und schuf in weiterer Folge Bedingungen, in denen „ein neues, national verortetes Begriffssystem an Gewicht gewann“. ${ }^{15}$

Im Zusammenhang mit der ,Revindikation“ Galiziens konstatiert Hans-Christian Maner beiden Monarchen einen deutlichen missionarischen Impetus. Schon bei Maria Theresia bestand das „Bild einer ehrerbietigen Provinz [...], deren Bewohner das aufklärerische Programm des Kaisers in sich aufgesogen hatten und sich regelrecht da-

9 Österreichisches Staatsarchiv, Allgemeines Verwaltungsarchiv (ÖStA, AVA). Hofkanzlei. Kt. 229: Pro Nota des Grafen von Pergen. 30.08.1772.

10 Larry Wolff: The Idea of Galicia. History and Fantasy in Habsburg Political Culture. Stanford 2010, S. 16.

11 Maner, Galizien (wie Anm. 8), S. 40.

12 Vgl. Horst Glassl: Das österreichische Einrichtungswerk in Galizien 1772-1790. München 1975, S. 87. 13 Vgl. Maner, Galizien (wie Anm. 8), S. 39-41. Darüber hinaus bestand auch eine grundsätzlich abschätzige Wahrnehmung des Adels und seiner ,polnischen Wirtschaft'. Vgl. Hubert Orłowski: „Polnische Wirtschaft“. Zum deutschen Polendiskurs in der Neuzeit. Wiesbaden 1996, S. 48.

14 Hans-Jürgen Bömelburg: Inklusion und Exklusion nach der Ersten Teilung Polen-Litauens. Die österreichische, preußische und russländische Regierungspraxis in Galizien, Westpreußen und den weißrussischen Gouvernements Polack und Mahilëŭ im Vergleich (1772-1806/07). In: Ders./Gestrich/ Schnabel-Schüle (Hg.): Die Teilungen Polen-Litauens (wie Anm. 1), S. 171-200, hier S. 196.

15 Ebd., S. 200. 
nach sehnten, ,erzogen zu werden““. ${ }^{16}$ Unter Josephs alleiniger Regentschaft ab 1780 erreichte diese Entwicklung ihren Höhepunkt, wollte der Kaiser Galizien doch mittels übereilter Reformen zur Musterprovinz umgestalten, wobei ihm laut Horst Glassl „der nüchterne Blick für das politisch Mögliche und das historisch Gewachsene“ fehlte. ${ }^{17}$ Während es schon zu Beginn kein Verwaltungskonzept, keine Richtlinien und darüber hinaus unklare Zuständigkeiten gegeben hatte, setzte sich diese uneinheitliche Entwicklung im josephinischen Jahrzehnt fort. ${ }^{18}$ Nichtsdestoweniger vollzog sich in dieser Zeit jedoch ein Prozess, den Larry Wolff als „cultural construction of Galician provincial identity“ bezeichnet. ${ }^{19}$ Die josephinische Publizistik, die in dem neuen Randgebiet „ein[en] Raum der Appropriations- und Zivilisierungsprojekte“ erkannte, ${ }^{20}$ schuf bis etwa 1790 unter Miteinbezug tradierter Polenstereotype die Basis für jenen Galiziendiskurs, der noch bis weit ins 19. Jahrhundert die Politik in der imperialen Peripherie entscheidend prägte.

\section{Die sprachliche Konzeption des neuen galizischen Schulwesens unter Maria Theresia}

Bereits Graf Pergen bemerkte nach der Inbesitznahme Galiziens, dass das dortige „Schul-Wesen und die Seminaria [...] in dem elendesten Stande“ seien, und empfahl die Einführung des in Erarbeitung befindlichen Normalschulsystems - nicht zuletzt, um der Bevölkerung „ein[en] ganz andere[n] Nationalgeist“ einzuflößen. ${ }^{21}$ Dieser Vorschlag nahm konkretere Gestalt an, als Maria Theresia die Einrichtung des galizischen Schulwesens der Studienhofkommission als oberster staatlicher Unterrichtsbehörde unterstellte und am 10. Mai 1774 deren Vorsitzenden, Baron von Kressel, anwies, die „Lage des dasigen Studien-Wesens“ $\mathrm{zu}$ sondieren und ein Gutachten zu erstellen, wie jenes ,auf den deutsch-erbländischen Fuß einzurichten seyn dörfte“. 22 Am 2. Januar 1775 erhielt das Gubernium in Lemberg schließlich die im Dezember des Vorjahres publizierte, von Johann Ignaz von Felbiger erarbeitete Allgemeine Schulordnung, für die deutschen Normal= Haupt= und Trivialschulen mit

16 Maner, Galizien (wie Anm. 8), S. 216.

17 Glassl, Einrichtungswerk (wie Anm. 12), S. 249.

18 Vgl. Bömelburg, Inklusion und Exklusion (wie Anm. 14), S. 181.

19 Wolff, The Idea of Galicia (wie Anm. 10), S. 14.

20 Larissa Cybenko: Ad imperii marginem. Die Natur Galiziens aus der Perspektive des Reisens in der Epoche des Josephinismus. In: Harald Haslmayr, Andrei Corbea-Hoişie (Hg.): Pluralität als kulturelle Lebensform. Österreich und die Nationalkulturen Südosteuropas. Wien-Berlin 2013 (Transkulturelle Forschungen an den Österreich-Bibliotheken im Ausland 8), S. 223-254, hier S. $251 \mathrm{f}$.

21 Zit. nach Anna Maria Harbig: Die aufgezwungene Sprache. Deutsch in galizischen Schulen (1772-1848). Białystok 2016, S. 45.

22 ÖStA, AVA. Studienhofkommission (StHK). Sign. 17. Kt. 79: Schreiben Maria Theresias an Baron Kreßel. 10.05.1774. 
dem Auftrag, einen entsprechenden Patententwurf für Galizien zu erarbeiten. ${ }^{23}$ Der daraufhin vom Gubernialrat Johann Christoph von Koranda erstellte Vorschlag kann als ,Grundlagenpapier' für die Einrichtung des staatlichen Schulwesens in der neuen Provinz gelten.

Für Koranda ergaben sich aus seinen Betrachtungen „über die Grundverfassung, Religion, und den National Charakter des Landes“ ${ }^{\text {24 } 4}$ entscheidende Fragen nicht nur in puncto der religiös-moralischen Erziehung, sondern auch in Bezug auf die Sprachenvielfalt der neuen Provinz. Dass Sprach- und Schulpolitik zu dieser Zeit bereits als eng miteinander verwoben erscheinen, lag in den bildungspolitischen

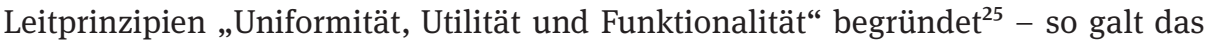
Wohl eines nach einheitlichen Grundsätzen verfassten habsburgischen Staatsverbandes als „übergeordnetes Leitziel“. ${ }^{26}$ Während die ,deutschen Schulen“ allen Untertanen „ein Mindestmaß an Elementarbildung“ vermitteln sollten, ${ }^{27}$ fiel dem höheren Schulwesen die Aufgabe zu, „das Deutsche als neue und unbestrittene Amts- und Kultursprache zu etablieren“. ${ }^{28}$ In seinen Ohnmaaszgebigen Gedanken betonte Koranda ebendiese Bedeutung des Deutschen, empfahl jedoch zugleich, nur in den größeren Städten Galiziens den deutschen Unterricht einzuführen schließlich war dessen flächendeckende Installation finanziell wie personell undurchführbar. ${ }^{29}$ Um diese Politik kulturell zu legitimieren, stellte Koranda in seinem Entwurf wiederholt eine zivilisatorische Rückständigkeit der neuen Provinz heraus laut Klemens Kaps ein entscheidendes „Dispositiv des Reformdiskurses“ im Josephinismus $^{30}$ - und wertete das Polnische ab. Felbigers Schulordnung folgend, hätte in den galizischen Volksschulen für die „römisch katholische Jugend“31 - die ruthenisch-unierte Bevölkerung wurde von Koranda de facto vom staatlichen Schulwesen ausgeschlossen - nämlich zweifelsfrei polnische Sprachlehre unterrichtet werden müssen. Koranda bemühte sich jedoch, das Polnische als hierfür ungeeignet

23 Vgl. Isabel Röskau-Rydel: Kultur an der Peripherie des Habsburger Reiches. Die Geschichte des Bildungswesens und der kulturellen Einrichtungen in Lemberg von 1772-1848. Wiesbaden 1993 (Studien der Forschungsstelle Ostmitteleuropa an der Universität Dortmund 15), S. 65.

24 ÖStA, AVA. StHK. Sign. 17. Kt. 79: Ohnmaaszgebige Gedanken. 1775.

25 Gerald Grimm: Expansion, Uniformisierung, Disziplinierung. Zur Sozialgeschichte der Schulerziehung in Österreich im Zeitalter des aufgeklärten Absolutismus. In: Wolfgang Schmale, Nan L. Dodde (Hg.): Revolution des Wissens? Europa und seine Schulen im Zeitalter der Aufklärung (1750 - 1825). Ein Handbuch zur europäischen Schulgeschichte. Bochum 1991, S. 225-254, hier S. 234.

26 Peter Haslinger: Sprachendynamik und imperiale Herrschaft in der Habsburgermonarchie 1740 - 1914. In: Zeitschrift für Ostmitteleuropa-Forschung 57/1 (2008), S. 81-111, hier S. $84 \mathrm{f}$.

27 Grimm, Sozialgeschichte der Schulerziehung (wie Anm. 25), S. 234.

28 Haslinger, Sprachendynamik (wie Anm. 26), S. 85.

29 Vgl. Harbig, Die aufgezwungene Sprache (wie Anm. 21), S. 49.

30 Klemens Kaps: Kulturelle Vorstellungswelten der Politischen Ökonomie. Bilder des habsburgischen Ostens im kameralistischen Diskurs zwischen den Wendejahren 1683 und 1815. In: Christoph Augustynowicz, Agnieszka Pufelska (Hg.): Konstruierte (Fremd-?)Bilder. Das östliche Europa im Diskurs des 18. Jahrhunderts. Berlin-Boston 2017, S. 179-199, hier S. 192.

31 Ohnmaaszgebige Gedanken (wie Anm. 24). 
herauszustellen. So referierte er auf die Kultivierung und Konsolidierung der deutschen Standardsprache, wie sie seit der Jahrhundertmitte auch im katholischen Süden erfolgt war, ${ }^{32}$ und stellte insbesondere die vermeintliche Sprachrichtigkeit und den Reichtum des Deutschen anhand seiner ,Literaturfähigkeit“ heraus. Demgegenüber erschien dem Schulreferenten die polnische Sprache mit ihrer angeblich starken dialektalen Gliederung als unterlegen. Indem Koranda sie „ein Gewebe von mehrerley Sprachen“ nannte, ${ }^{33}$ sprach er das im zeitgenössischen Denken vernichtende Verdikt der ,Mischsprache“ aus und verweigerte ihr das zentrale ,Gütesiegel ${ }^{\text {‘ }}$ der sprachlichen ,Reinheit‘. ${ }^{34}$ Resümierend empfahl er daher, „die Lehrer der Normalschule mit der Sprachlehre der Muttersprache zu verschonen“. ${ }^{35}$ Nun dürfen die Ohnmaaszgebigen Gedanken nicht dahingehend missverstanden werden, dass Koranda das Polnische gänzlich aus den Schulen verbannen wollte - immerhin betonte der Schulreferent explizit die Notwendigkeit von Schulbuchübersetzungen. Allerdings zielte die Weigerung zur Abfassung einer polnischen Schulgrammatik bei gleichzeitig nicht vorgesehenem Einsatz ausländischer Lehrmaterialien laut Anna Maria Harbig darauf ab, „die Muttersprache auf einfachste Kommunikation zu beschränken und deren Entfaltung als Medium des Denkens, als Träger von Kultur und Identität zu verhindern“. ${ }^{36}$ Dafür spricht auch die Tatsache, dass in weiterer Folge hauptsächlich zweisprachige Lehrer aus Böhmen und Mähren als galizische Lehrkräfte angeworben wurden, man es also für ausreichend befand, ein dem Polnischen „verwandtes Idiom“ $\mathrm{zu}$ beherrschen. ${ }^{37}$

In Wien wurde der Patententwurf des Lemberger Schulreferenten zunächst von der Niederösterreichischen Schulkommission diskutiert, der mit Felbiger auch der Verfasser der Allgemeinen Schulordnung angehörte. In einer Sitzung am 15. Mai 1775 zeigte man sich mit den Vorschlägen in großen Teilen einverstanden, lediglich die Ausklammerung der ruthenischen Bevölkerung vom neuen Schulwesen hielt man für „bedenklich“. ${ }^{38}$ Kritisiert wurde zudem die Empfehlung Korandas, „die deutsche

32 Vgl. Peter von Polenz: Deutsche Sprachgeschichte vom Spätmittelalter bis zur Gegenwart. Bd. 2: 17. und 18. Jahrhundert. Berlin-New York 1994, S. $168 \mathrm{f}$.

33 Ohnmaaszgebige Gedanken (wie Anm. 24).

34 Diese Bemerkungen sind im Kontext einer aufklärerischen Sprachkritik zu lesen, die sich gegen „die als modisch-affektiert oder scheingelehrt und daher unnötig empfundene Verwendung von Fremdwörtern“ richtete. Andreas Gardt: Nation und Sprache in der Zeit der Aufklärung. In: Ders. (Hg.): Nation und Sprache. Die Diskussion ihres Verhältnisses in Geschichte und Gegenwart. Berlin-New York 2000, S. $169-198$, hier S. 183.

35 Ohnmaaszgebige Gedanken (wie Anm. 24).

36 Harbig, Die aufgezwungene Sprache (wie Anm. 21), S. 56.

37 Ulrike Eder: „Auf die mehrere Ausbreitung der teutschen Sprache soll fürgedacht werden“. Deutsch als Fremd- und Zweitsprache im Unterrichtssystem der Donaumonarchie zur Regierungszeit Maria Theresias und Josephs II. Innsbruck u. a. 2006 (Österreichische Beiträge zu Deutsch als Fremdsprache B 9), S. 132.

38 ÖStA, AVA. StHK. Sign. 17. Kt. 79: Protokollsauszug der N. Ö. Schulkommission. 15.05.1775. Da Korandas diesbezügliche Vorschläge in Wien keine wesentlichen Änderungen mehr erfuhren, handelte es sich hierbei um eine folgenreiche bildungspolitische Weichenstellung. 
Sprache ausser zu Lemberg und in den Gymnasien auf dem Lande nicht [zu lehren]“, musste es doch

unstrittig zur Verbesserung und Verfeinerung der Sitten und zur Abschleifung der rauhen Gedenkensart sehr vieles beytragen, wenn die deutsche Sprache dort Landes unter den Innwohnern aller Stände mehr verbreitet wird: Selbsten in die politische Verfassung kann dieses einen starken Einfluß haben, indem es dazu beyträgt, daß diese nunmehr unter deutscher Beherrschung stehende Nation vielmehr Neigung zu solcher gewinne [...]. ${ }^{39}$

Auffällig ist an dieser Begründung nicht bloß der klar herausgestellte Konnex zwischen Sprache und Denken sowie Sittlichkeit, sondern auch die Reihung der Argumente, erscheint doch die utilitaristische Vorstellung von der einheitlichen Staatssprache klar als zweitrangig. Man gewinnt gar den Eindruck, als wäre der Einfluss der erörterten sprachpolitischen Maßnahmen auf „die politische Verfassung“ nur ein willkommener Nebeneffekt des zivilisatorischen Sendungsbewusstseins der neuen Staatsmacht - subjektive Rechtfertigung und objektiver Zweck klaffen also merklich auseinander. Nach dieser Klarstellung, dass es sehr wohl langfristiges Ziel der staatlichen Schulpolitik sein müsste, das Deutsche auch in den ländlichen Gebieten Galiziens zu verbreiten, besann man sich letztlich jedoch auf den von Koranda geäußerten Pragmatismus, konkrete Maßnahmen vorerst nur im städtischen Raum zu treffen. Felbiger, der in einem separat eingereichten Votum Kritik an den Vorschlägen des Schulreferenten übte, ${ }^{40}$ konnte sich mit seinem Plädoyer für das Lehrziel der polnischen Sprachlehre an den galizischen Volksschulen nicht durchsetzen.

Es sollte noch fast zwei Jahre dauern, bis die Hofkanzlei der Kaiserin den Vorschlag zur Einführung des Normalschulwesens in Galizien vorlegte. Bezeichnenderweise wurde auch 1777 Korandas Empfehlung, wonach die „Muttersprache, namlich die pohlnische derzeit nicht gelehret werden [könne], weil sie nicht in sichere Regeln gebracht wäre, und in verschiedenen Provinzen Pohlens anders gesprochen werde“, noch kommentarlos wiederholt, um zu resümieren, dass „auf die Verbesserung der polnischen Sprache kein sonderlicher Bedacht zu nehmen seyn dürfte“. ${ }^{41}$ Was den polnischen Adel betraf, so ging die Absicht der Hofkanzlei dahin, „bey demselben vielmehr die teutsche Sprache nach und nach so viel immer thunlich gemein zu machen“, und man betonte, dass „seit der Revindication der Gebrauch der pohlnischen Sprache [...] bereits gänzlich abgestellet worden“ sei. ${ }^{42}$ Nach dieser Bagatellisierung der Stellung des Polnischen und der Beschönigung der sprachlichen Zustände in Galizien machte die Anregung von Schulbuchübersetzungen offenkundig keinen großen Eindruck auf die Kaiserin, die unter dem Vortrag notierte:

39 Ebd.

40 Vgl. ÖStA, AVA. StHK. Sign. 17. Kt. 79: Votum des Saganischen Prälaten. 1775.

41 ÖStA, AVA. StHK. Sign. 17. Kt. 79: Vortrag der Hofkanzlei. Wien, 15.03.1777.

42 Ebd. 
Ich bin in allen mit diesen wohl verfasten Plan verstanden das die Sache nur keinen Auffenthalt mehr bekome. Wegen Übersetzung in die pohlnische Sprach bin nicht so pressirt das vor allen die teutsche Sprach alda solle vervielfältigt werden [...]. ${ }^{43}$

Letztlich konnte sich aber auch Maria Theresia nicht gegen diese Notwendigkeit stellen, zumal die Zeit drängte - bereits im Herbst 1775 hatte die Lemberger Normalschule ihren Betrieb aufgenommen. ${ }^{44}$ Am 22. März 1777 wurde der Patententwurf daher nach Lemberg gesandt, wo er bis 1805 als galizische Schulordnung diente..$^{45}$ Indes stellte sich bereits bei der Gründung der Normalschule, die als ,Musterschule“ der Provinz auch zur Ausbildung weiterer Lehrer angedacht war, der eklatante Personalmangel als größtes Problem der staatlichen Schulpolitik dar. Gemäß den üblichen Schritten - „Bestellung von Schulkommissionen, Einrichtung von Normalschulen, Ausbildung von Lehrern und Ausbau des Schulnetzes“46 - wurde auch in Galizien per Hofdekret vom 27. März 1776 eine eigene Schulkommission gegründet, ${ }^{47}$ deren Tätigkeit aufgrund personeller Überforderung aber überschaubar war. Bis 1780 blieb die Lemberger Normalschule die einzige neu eingerichtete Schule in der ganzen Provinz, ${ }^{48}$ und Joseph II. zeigte sich auf seiner zweiten Galizienreise im selben Jahr enttäuscht über den langsamen Aufbau des Schulwesens. ${ }^{49}$ Als Graf von Gallenberg, der Leiter der Schulkommission, noch 1783 berichtete, dass bislang nur in Lemberg die Schulen „eine zweckmäßigere Leitung“ erhalten hätten, ${ }^{50}$ befahl der Kaiser kurzerhand die Aufhebung der „dem Schulwesen mehr schädlich als nützlich gewesenen Studien Commission“..$^{51}$

Wie bereits angeklungen, legte die theresianische Schulpolitik ihren Fokus auf den Aufbau des staatlichen Elementarschulwesens. Dieser Schwerpunkt im primären Bildungssektor muss im Kontext einer „sozial restriktive[n] Bildungspolitik“ betrachtet werden, die den Wandel des Gymnasiums „,von der reinen, prinzipiell allen sozialen Schichten zugänglichen Ordensinstitution [...] zur staatlichen, sozial exklusiven Bildungsinstitution" vollzog. ${ }^{52}$ In sprachpolitischer Hinsicht ist hierbei vor allem der 1775 eingeführte Lehrplan des Piaristen Gratian Marx von Bedeutung, der dem Deutschen eine Vorzugsstellung an den Gymnasien einräumte ${ }^{53}$ und zu-

43 Ebd.

44 Vgl. Eder, Deutsch als Fremd- und Zweitsprache (wie Anm. 37), S. 128.

45 Vgl. Harbig, Die aufgezwungene Sprache (wie Anm. 21), S. 46.

46 Helmut Engelbrecht: Geschichte des österreichischen Bildungswesens. Erziehung und Unterricht auf dem Boden Österreichs. Bd. 3: Von der frühen Aufklärung bis zum Vormärz. Wien 1984, S. 132.

47 Vgl. ÖStA, AVA. StHK. Sign. 1. Kt. 1: Dekret an das Gubernium. Wien, 27.03.1776.

48 Vgl. Glassl, Einrichtungswerk (wie Anm. 12), S. $237 \mathrm{f}$.

49 Vgl. Röskau-Rydel, Kultur an der Peripherie (wie Anm. 23), S. $66 \mathrm{f}$.

50 Zit. nach ebd., S. 66.

51 ÖStA, AVA. StHK. Sign. 1. Kt. 1: Dekret an das Gubernium. 11.12.1783. Der Begriff „Studien Commission" meint hier die galizische Schulkommission.

52 Grimm, Sozialgeschichte der Schulerziehung (wie Anm. 25), S. $236 \mathrm{f}$.

53 So sollte der Unterricht in den ersten beiden ,Grammatical-Klassen' ausschließlich auf Deutsch stattfinden. Vgl. Harbig, Die aufgezwungene Sprache (wie Anm. 21), S. 79. 
gleich den Lateinunterricht als Hauptlehrgegenstand zementierte. ${ }^{54}$ Im Gegensatz zum kaum vorhandenen Volksschulwesen gab es 1772 in Galizien verhältnismäßig viele höhere Schulen für die Söhne der polnischen Adeligen. ${ }^{55}$ Auch hier gewann das Deutsche anfänglich durch die Machtkonzentration hin zur neuen Regierung an Bedeutung, während zur Förderung des Polnischen keine besonderen Maßnahmen getroffen wurden - schließlich war der Unterricht in den Volkssprachen, die zur einfachen Kommunikation innerhalb der Landbevölkerung dienen sollten, nur als pragmatische Zwischenlösung konzipiert. ${ }^{56}$

In der Hauptstadt Lemberg sollte die Umwandlung der galizischen Gymnasien gewissermaßen als Pilotprojekt ihren Anfang nehmen, weshalb mit 1. September 1778 nach Maßgabe des Guberniums „die Humaniora [...] vollkommen deutsch und lateinisch, und nicht mehr pohlnisch lateinisch tradiret“" und zudem keine Schüler ohne Normalschulzeugnis zum Gymnasium zugelassen werden sollten. ${ }^{57}$ Neben deutschen Professoren erbat das Gubernium hierfür vom Aufklärungspädagogen Karl Heinrich Seibt auch die „Abschickung eines tüchtige[n] Lehrer[s]“ aus Prag (Praha), um den Schülern durch die Vermittlung des deutschen Schreibstils und der belles lettres „die vornehmsten Punkte anzuzeigen, woraus sie um richtig und edel zu denken folglich auch gut zu schreiben [haben] und ihre Denkungs Art läuteren müssen“ “. ${ }^{58}$ Aus diesem Schreiben geht deutlich hervor, dass die ,rohen“ Landessitten der Integration Galiziens in die Gesamtmonarchie nach Meinung der aufgeklärt-bürgerlichen Beamtenschaft mindestens ebenso entgegenstanden wie die sprachliche Situation in der neuen Provinz. In Wien bremste man die Pläne des Guberniums jedoch wiederholt aus und drängte auf eine bloß „successive Besetzung der lateinischen Lehr Kanzeln mit teutschen Professoribus“.59 Dies mochte zum einen im bereits angeklungenen Lehrermangel begründet sein, zum anderen wurde aber auch die Befürchtung geäußert, dass durch die überhastete Einführung des deutschen Unterrichts „die Nation vielleicht auf den Irwahn gerathen könte, als ob man ihre Muttersprache, oder die ihnen so angenehme lateinische Sprache gänzlich ausrotten wolte“ ${ }^{60}$ Die Verbreitung des Deutschen im sekundären Bildungssektor schritt in den Folgejahren jedenfalls nur in bescheidenem Maß voran, wobei auch im Gymnasialbereich jegliche Reformbestrebungen der neuen Regierung durch den chronischen Personalmangel ausgebremst wurden - auf

54 Vgl. Grimm, Sozialgeschichte der Schulerziehung (wie Anm. 25), S. 246.

55 Vgl. Glassl, Einrichtungswerk (wie Anm. 12), S. 238.

56 Vgl. Katharina Weisswasser: Die Sprachenpolitik der Habsburger gegenüber den Polen in Galizien. In: Ewa Cwanek-Florek, Irmgard Nöbauer (Hg.): Sprachliche Wechselbeziehungen in der Habsburgermonarchie. Wien 2015 (Symposien und Seminare am Wissenschaftlichen Zentrum der Polnischen Akademie der Wissenschaften in Wien 13), S. 19-40, hier S. 21.

57 ÖStA, AVA. StHK. Sign. 10. Kt. 59: Bericht des Guberniums. Lemberg, 09.12.1775. Der Elementarunterricht sollte also auf die reibungslose Einführung des deutschen Unterrichts im sekundären Bildungssektor hinarbeiten.

58 Ebd. Vgl. zu Seibt auch den Beitrag von Ivo Cerman in dieser Themenausgabe.

59 ÖStA, AVA. StHK. Sign. 10. Kt. 59: Dekret an das Gubernium. Wien, 18.05.1776.

60 Ebd. 
nicht deutschsprachige Ordensgeistliche, die die galizischen Schuleinrichtungen bisher geleitet hatten, konnte man daher nie gänzlich verzichten. ${ }^{61}$

\section{Verschärfter Sprachzwang und Schulausbau unter Joseph II.}

Auf den Herrschaftsantritt Josephs II. in den Erblanden folgte eine radikale Unifizierungspolitik, die in der heterogenen Habsburgermonarchie naturgemäß im Bereich der Sprachenfrage ihren pointierten Ausdruck finden musste. Deutlich stärker als bei Maria Theresia spiegelte sich in der Person des aufgeklärt-absolutistisch agierenden Herrschers der „Zusammenhang zwischen imperialer Herrschaft und sprachpolitischem Gestaltungsprimat wider“, wobei Joseph das Ziel verfolgte, „die Gesamtstaatlichkeit aller Teile der Habsburgermonarchie vorrangig durch eine gemeinsame Amtssprache, das Deutsche, voranzutreiben“. ${ }^{62}$ Ab 1780 überschlugen sich die zumeist zum Scheitern verurteilten Reformen, die nur das künftige Ziel vor Augen hatten, jedoch „ohne Rücksicht auf die bestehenden Verhältnisse und die finanziellen Möglichkeiten in Angriff genommen wurden“.63 Im Gegenteil wurde das Deutsche als „Sprache der Macht“ vor allem in den östlichen Gebieten der Monarchie zunehmend als „Sprache der Unterdrücker“ erlebt. ${ }^{64}$ Wenngleich die in jener Zeit aufkommenden sprachnationalen Konzepte in ihrer politischen Wirkung noch marginal blieben, so kam es doch infolge der josephinischen Reformpolitik zu einer „Mobilisierung der Peripherien gegen die Dynamik kultureller, politischer und sprachlicher Normierung durch das imperiale Zentrum“, wobei die Erweckung eines sprachpolitischen Bewusstseins als „nicht intendierte Folgewirkung von Sprachreform, Bildungspolitik und weitgehender Pressefreiheit“ betrachtet werden muss. ${ }^{65}$

In Galizien verband sich das Vordringen der deutschen Sprache noch deutlicher als zuvor mit den auf bildungspolitischem Gebiet eingeleiteten Modernisierungsmaßnahmen. Während der als ,National-Erziehung، verstandene Deutschunterricht in den Fokus rückte, verschob sich gleichzeitig das sprachpolitische Interesse immer stärker von den Gymnasien hin zu den Volksschulen. ${ }^{66}$ Den Startschuss für die Expansion des staatlichen Elementarschulwesens bildete die 1783 erfolgte Auflösung der von Joseph für unzulänglich befundenen galizischen Schulkommission, um „demnach in diesem höchstwichtigen und zur Bildung und Umstaltung der Nation unumgänglich nöthigen Geschäfte die Zeit nicht ferner [...] unthatig zu verlieren“. ${ }^{67}$

\footnotetext{
61 Vgl. Röskau-Rydel, Kultur an der Peripherie (wie Anm. 23), S. 149.

62 Haslinger, Sprachendynamik (wie Anm. 26), S. 87.

63 Glassl, Einrichtungswerk (wie Anm. 12), S. 249.

64 Eder, Deutsch als Fremd- und Zweitsprache (wie Anm. 37), S. $126 \mathrm{f}$.

65 Haslinger, Sprachendynamik (wie Anm. 26), S. $88 \mathrm{f}$.

66 Vgl. Harbig, Die aufgezwungene Sprache (wie Anm. 21), S. $76-78$.

67 ÖStA, AVA. StHK. Sign. 17. Kt. 79: Dekret an das Gubernium. 11.12.1783.
} 
1784 wurde Johann Nepomuk Franz Hofmann, seit 1778 Normalschuldirektor in Lemberg, vom Kaiser zum Normalschulenoberaufseher in Galizien ernannt. ${ }^{68}$ Er trieb den Aufbau des Schulwesens in der Provinz nach Kräften voran und konnte bereits im selben Jahr Schulgründungen in einigen Kreisstädten erreichen. ${ }^{69}$ Vorerst blieb Galizien jedoch auch weiterhin auf zugezogene Lehrer aus Böhmen und Mähren sowie Schlesien und dem Ermland angewiesen. ${ }^{70}$ Zudem waren, wie aus einer Prüfungseinladung aus dem Jahr 1784 hervorgeht, ${ }^{71} \mathrm{zu}$ jenem Zeitpunkt offenbar noch immer keine offiziellen polnischen Schulbuchübersetzungen angefertigt worden, obgleich auch der böhmische Schulreformer Ferdinand Kindermann diese Notwendigkeit betonte, da sich „die Aufklärung auf das Land ausbreiten müsse“. ${ }^{72}$ Kindermann riet überdies zur besseren Ausbildung der Geistlichen, die als Landschullehrer eine Schlüsselrolle in der Reform des galizischen Schulwesens spielen müssten - noch sei nämlich die dortige Geistlichkeit „sehr wenig zu brauchen, und überhaupt bey dieser Nation ein sehr geringes Licht verbreitet“ ${ }^{73}$

Seitens der Regierung ging man in der Folge daran, die Priesterseminare unter dem Gesichtspunkt der Volksaufklärung umzugestalten, wobei das Gubernium angewiesen wurde, den Gebrauch des Deutschen „besonders in den General Seminarien zu befördern“.${ }^{74}$ All jene Maßnahmen mussten jedoch früher oder später an der Unzulänglichkeit des galizischen Schulfonds scheitern, die dazu führte, dass 1786 der Ausbau der ländlichen Trivialschulen zwischenzeitlich sogar abgebrochen wurde. ${ }^{75}$ Im Rahmen einer am 28. Juni 1787 beim Gubernium zusammengetretenen Kommission, bei der schulpolitische Zielsetzungen und Probleme erörtert wurden, kritisierte der Vizepräsident Baron von Margelik den schleppenden Aufbau des Schulwesens. So bestanden in ganz Galizien erst 144 Schulen mit etwas über 7.800 Schülern, während das Gubernium die Gesamtzahl unterrichtsfähiger Kinder auf 500.000 schätzte $^{76}$ und 1789 eine Zahl von 2.500 nötigen Pfarrschulen ermittelte, um den Bedarf des Landes zu decken. ${ }^{77}$ Offenbar schritt der Ausbau des Schulwesens nur in den verstreuten

68 Vgl. Röskau-Rydel, Kultur an der Peripherie (wie Anm. 23), S. 67.

69 Vgl. Glassl, Einrichtungswerk (wie Anm. 12), S. 240.

70 Vgl. Isabel Röskau-Rydel: Zwischen Akkulturation und Assimilation. Karrieren und Lebenswelten deutsch-österreichischer Beamtenfamilien in Galizien (1772-1918). München 2015 (Schriften des Bundesinstituts für Kultur und Geschichte der Deutschen im östlichen Europa 55), S. 82.

71 Vgl. ÖStA, AVA. StHK. Sign 18. Kt. 92: Einladung zur öffentlichen Prüfung. Lemberg, 1784.

72 ÖStA, AVA. StHK. Sign. 17. Kt. 79: Vortrag der Studienhofkommission. Wien, 26.01.1785. Eine erste Ausgabe des Elementarz, der polnischen Übersetzung des auf Felbiger zurückgehenden Nahmenbüchleins, ist erst für 1789 nachweisbar. Vgl. Harbig, Die aufgezwungene Sprache (wie Anm. 21), S. 237. 73 Vortrag der Studienhofkommission vom 26.01.1785 (wie Anm. 72).

74 ÖStA, AVA. StHK. Sign 17. Kt. 79: Schreiben an das Gubernium. Wien, 09.09.1787.

75 Vgl. Harbig, Die aufgezwungene Sprache (wie Anm. 21), S. 84.

76 Vgl. ÖStA, AVA. StHK. Sign 17. Kt. 79: Protokoll einer beim Gubernium abgehaltenen Kommission. 28.06.1787.

77 Vgl. ÖStA, AVA. StHK. Sign 17. Kt. 79: Protokoll einer beim Gubernium abgehaltenen Kommission. 17.01.1789. 
deutschen Kolonien vergleichsweise zügig voran. Wie aus dem Protokoll vom 28. Juni 1787 hervorgeht, maß der Schulenoberaufseher Hofmann der „bessere[n] Bestellung der Kolonieschulen“ wohl eine überproportional hohe Bedeutung bei. ${ }^{78}$ Das Motiv für diese Förderung war vor dem Hintergrund einer Verbreitung des Deutschen in der Provinz evident: Schließlich könnte beim Anwerben von Lehrern „auf einige des Lesens, und Schreibens kündige deutsche Landleute der vorzügliche Bedacht genommen“ werden. ${ }^{79}$ Auch der umtriebige Gubernialrat Ernst Traugott von Kortum maß den neuen Ansiedlungen für die Verbreitung der deutschen Sprache große Bedeutung bei. Allerdings scheint die Vorstellung einer Zivilisierungsmission in Galizien 1787 schon einer gewissen Ernüchterung, respektive gar einer Angst vor dem nachteiligen Einfluss des neuen Lebensumfelds auf die geistige wie ökonomische Produktivität der Kolonisten gewichen zu sein, befürchtete Kortum doch, dass „der Deutsche sich nach und nach den Sitten, Sorglosigkeit, und der sklavischen Denkart des Nazionalen beigeselle[n]“ könnte. ${ }^{80}$ Die offensichtlichen Schwierigkeiten beim Aufbau eines auf die Verbreitung des Deutschen ausgelegten staatlichen Schulwesens begegnen in den Berichten des Guberniums während des gesamten josephinischen Jahrzehnts wiederholt. So hatte man schon 1784 beklagt,

daß die weite Ausdehnung des Landes, die gänzliche Rohheit des gemeinen Volkes [...], die Vorurtheile der Obrigkeiten, und Herrschaften, und hundert andere diesem Lande eigenen Hinderniße das Geschäft der Schuleinrichtungen aller Art in Gallizien zu einem weit schwierigeren Gegenstand als in den übrigen deutschen Erblanden machen. In diesen ist nur zu verbeßeren und vollkommener zu machen, hier hingegen muß alles neu geschehen, und das alte zum Theil ausgerottet werden $[. . .]^{81}$

Der Kaiser zeigte sich von derlei Schilderungen jedoch konsequent unbeeindruckt und kritisierte wiederholt die vermeintliche Untätigkeit seiner Beamten. Noch 1788 dekretierte er, „dass in allen Städten und auf dem Lande [...] der Unterricht in deutscher Sprache ertheilet werden solle“82 - eine Vorgabe, die für Galizien nie umgesetzt werden konnte.

Bereits 1781 weitete Joseph II. die Normalschulpflicht auch auf die Juden aus, ${ }^{83}$ wobei die kaiserliche Schulpolitik von Beginn an emanzipative und repressive Züge in sich vereinte und die für die sprachpolitischen Maßnahmen in Galizien so charakteristische Verschränkung von Anreiz und Repression auf die Spitze trieb. Ziel war die „sittliche Bildung und Aufklärung der Juden“ durch eine Ersetzung des Hebräischen

78 Kommissionsprotokoll vom 28.06.1787 (wie Anm. 76).

79 Ebd.

80 ÖStA, AVA. StHK. Sign 17. Kt. 79: Auszug aus einem Vortrag von Hofkanzlei, Hofkammer und Bankodeputation. 10.07.1787.

81 ÖStA, AVA. StHK. Sign 10. Kt. 57: Bericht des Guberniums. Lemberg, 05.05.1784.

82 Zit. nach Harbig, Die aufgezwungene Sprache (wie Anm. 21), S. 85.

83 Vgl. Dirk Sadowski: Haskala und Lebenswelt. Herz Homberg und die jüdischen deutschen Schulen in Galizien 1782-1806. Göttingen 2010 (Schriften des Simon-Dubnow-Instituts 12), S. 68. 
sowie des Jiddischen durch das Deutsche. ${ }^{84}$ Während die Errichtung der Schulen anfangs noch in Absprache mit den jeweiligen Kahalen, den traditionellen jüdischen Gemeinden, erfolgte, nahm die josephinische Judenpolitik mit der Einführung des Schulzwangs ab 1785 zunehmend repressive Züge an, weshalb die in weiten Teilen konservative jüdische Bevölkerung Galiziens den neuen Schulen ablehnend gegenüberstand. ${ }^{85}$ Auch der 1787 eigens angestellte jüdische Schulenoberaufseher Herz Homberg war nicht in der Lage, den Widerstand der orthodoxen Juden „mit psychologisch geschickt gewählten Mitteln abzubauen“, und setzte stattdessen verstärkt auf die Staatsgewalt. ${ }^{86}$ Dass der Chassidismus unter den galizischen Juden an Boden gewann, war dabei nur einer der Problemkomplexe, denen sich Homberg gegenübersah, stand doch der Großteil der Lemberger Beamtenschaft dem Schulprojekt aufgrund antijüdischer Ressentiments von Beginn an ablehnend gegenüber.

Der Unterricht an den staatlichen jüdischen Schulen unterschied sich auf den ersten Blick nur geringfügig von jenem an den christlichen Trivialschulen Galiziens. Das Spezifikum bestand offiziell lediglich darin, dass die dort für den Religionsunterricht vorgesehenen Unterrichtseinheiten in den jüdischen Schulen stattdessen zur „Übung in dem schriftlichen Aufsatze, und der Sprachlehre“ verwendet werden sollten, ${ }^{87}$ das Curriculum also stärker auf die Vermittlung deutscher Sprachkenntnisse zugeschnitten war. Allerdings rührte aus der Vorstellung von einer besonderen sittlich-moralischen ,Verdorbenheit' der Juden „ein spezifischer Impetus des Unterrichts“. ${ }^{88}$ Im Gegensatz zum christlichen Curriculum firmierte etwa der deutsche Leseunterricht auch unter der Bezeichnung „Moralunterricht“, 89 erschienen sprachliche und sittliche Erziehung also besonders stark aneinander gebunden. Dass die Verdrängung des Jiddischen als innerjüdisches Kommunikationsmittel das erklärte Unterrichtsziel darstellte, wird aus den offiziellen Verhaltungspunkten für die jüdischen Lehrer Galiziens deutlich, in denen sie explizit angewiesen wurden, „der jüdischen Schuljugend die ächte Aussprache der deutschen Mundart beizubringen, und die unter Juden üblichen deutschpohlnischen sprachwidrigen Redensarten anzumerken“. ${ }^{90}$ Die aus diesen Vorgaben resultierende „Herrschsucht“ der Lehrer wurde denn auch unmittelbar nach dem Tod Josephs II. in einer Bittschrift der Gemeindevertreter

84 Ebd., S. 71.

85 Vgl. ebd., S. 110 - 118.

86 Wolfgang Häusler: Das galizische Judentum in der Habsburgermonarchie. Im Lichte der zeitgenössischen Publizistik und Reiseliteratur von 1772-1848. Wien 1979, S. 43. Vgl. zu Homberg auch den Beitrag von Armin Langer in dieser Themenausgabe.

87 ÖStA, AVA. StHK. Sign. 23. Kt. 106: Schreiben der Studienhofkommission an das Gubernium. Wien, 16.10.1787.

88 Sadowski, Haskala und Lebenswelt (wie Anm. 83), S. $167 \mathrm{f}$.

89 Ebd., S. 184.

90 ÖStA, AVA. StHK. Sign. 23. Kt. 106: Verhaltungspunkte für jüdische Schullehrer in Galizien und Lodomerien [vermutlich 1791]. 
an Kaiser Leopold beklagt, ${ }^{91}$ wobei die Unzufriedenheit der jüdischen Bevölkerung mit den staatlichen Bildungseinrichtungen letztlich dazu führte, dass sie 1806 aufgelöst und mit den allgemeinen Volksschulen vereinigt wurden. ${ }^{92}$

Die Entwicklungen im Bereich sekundärer Bildung standen unter der Herrschaft Josephs II. ebenfalls ganz im Zeichen einer zunehmenden Vereinnahmung durch den Staat. Am bedeutsamsten erwies sich hierbei die Reduktion der galizischen Lateinschulen auf sechs und deren Umwandlung in staatliche Gymnasien. Am 11. Dezember 1783 wurde dem Gubernium die schon unter Maria Theresia erwogene Reduktion dekretiert, ${ }^{93}$ während die Eröffnung der neuen staatlichen Gymnasien für September 1784 beschlossen wurde. ${ }^{94}$ Da Wien zeitgleich mit dieser Umstrukturierung auch verordnete, dass „ohne besondere Erlaubniß des Guberniums kein Landeskind auf auswärtige Schulen und Universitäten geschickt werden solle, “95 wurde der traditionelle Bildungsweg des polnischen Adels auf einen Schlag nachhaltig verändert. ${ }^{96}$ Gerade der hohe Anteil polnischer Geistlicher am Lehrkörper sollte sich jedoch als größtes Hindernis für die Durchsetzung des neuen Lehrplans erweisen. Als das Gubernium im September 1786 nämlich nach Wien Bericht erstattete, musste man feststellen, dass mit Ausnahme des Lemberger Gymnasiums nach wie vor auf Polnisch und Lateinisch gelehrt wurde, „weil der größte Theil der Professoren Nationalisten“ waren. ${ }^{97}$ Erwartbar negativ fiel denn auch das Resümee der Studienhofkommission aus, für die der Bericht „die lang wahrgenommenen Gebrechen an den dortigen Gymnasien“ bestätigte. Neben dem Problemfaktor Sprache wurden vor allem auch „Vorurtheile“ kritisiert, welche die für unaufgeklärt befundenen Lehrer an die Schüler weitervermittelten, und die „sehr nachtheilige Spuren und Folgen in der künftigen Denkensart zurücklassen“ müssten. ${ }^{98}$ Die Erfolge der staatlichen Sekundarschulpolitik waren also auch im josephinischen Jahrzehnt überschaubar. Nichtsdestotrotz wurde die repressive Sprachpolitik gegen das Polnische noch bis zur Mitte des 19. Jahrhunderts fortgesetzt. ${ }^{99}$

91 ÖStA, AVA. StHK. Sign. 23. Kt. 106: Schreiben der Deputierten sämtlicher Judengemeinden Galiziens an Leopold II. Wien, 03.08.1790.

92 Vgl. Röskau-Rydel, Kultur an der Peripherie (wie Anm. 23), S. $117 \mathrm{f}$.

93 Vgl. ÖStA, AVA. StHK. Sign. 10. Kt. 57: Dekret an das Gubernium. 11.12.1783.

94 Vgl. Eder, Deutsch als Fremd- und Zweitsprache (wie Anm. 37), S. 214.

95 Dekret vom 11.12.1783 (wie Anm. 93).

96 Vgl. Harbig, Die aufgezwungene Sprache (wie Anm. 21), S. 80 f.

97 ÖStA, AVA. StHK. Sign. 10. Kt. 57: Bericht des Guberniums über den Zustand der Gymnasien. Lemberg, 28.09.1786. Der Begriff ,Nationalisten“ meint hier Einheimische.

98 ÖStA, AVA. StHK. Sign. 10. Kt. 57: Vortrag der Studienhofkommission über den Zustand der Gymnasien in Galizien. Wien, 29.11.1786.

99 Vgl. Röskau-Rydel, Kultur an der Peripherie (wie Anm. 23), S. 150 f. 


\section{Die Folgen der josephinischen Sprach- und Schulpolitik in Galizien}

In der Habsburgermonarchie hatte sich bereits in den letzten Regierungsjahren Josephs II. eine Krise des ,Aufgeklärten Absolutismus‘ immer deutlicher abgezeichnet. ${ }^{100}$ Die Spannungen zwischen Reformoptimismus und den Erfordernissen absolutistischer Herrschaft gingen auch an Galizien nicht spurlos vorüber und manifestierten sich unmittelbar nach dem Tod des Kaisers in zwei Memoranden des polnischen Adels, die dessen Forderungen betreffend eine Neuregelung grundlegender Fragen auch des Schulwesens sowie des öffentlichen Sprachgebrauchs zum Ausdruck brachten. ${ }^{101}$ Die Charta Leopoldina, die in sprachpolitischer Hinsicht auf eine partielle Restitution des Lateinischen sowie verstärkten Gebrauch des Polnischen zulasten des Deutschen abzielte, vereinigte dabei konservative und progressive Forderungen gleichermaßen ${ }^{102}$ und spiegelt bereits die einsetzende politische Instrumentalisierung von Sprachenfragen wider. Der Lemberger Gubernialrat Kortum, der in einer Publikation gegen den Adel polemisierte, ${ }^{103}$ war dabei nicht der einzige, der die Charta als Gefahr für das habsburgische Zivilisierungsprojekt wahrnahm. Eine zu Jahresbeginn 1791 zusammengetretene Kommission sah das Problem grosso modo in einer misslungenen Integration des Adels in den Gesamtstaat. Dessen sprachpolitische Forderungen lehnte man durchweg ab und betonte insbesondere den Konnex zur Schulpolitik, gingen doch mit der geforderten Aufhebung der deutschen Geschäftssprache

auch alle deutschen Schulanstalten, mithin auch alle Aufklärung zu Grunde [...]. Des Nachtheils will man gar nicht gedenken, den die Aufklärung schon dadurch litte, daß die deutsche Sprache in dem Unterrichte durch die polnische, vor welcher sie durch jede Art von Kultur so unendlich viel voraus hat, ersezt werden müßte. ${ }^{104}$

100 Vgl. Helmut Reinalter: Joseph II., der Josephinismus und die Aufklärung. In: Ders. (Hg.): Selbstbilder der Aufklärung. Innsbruck u.a. 2007 (Interdisziplinäre Forschungen 20), S. 45-55, hier S. $49-54$.

101 Vgl. Wolff, The Idea of Galicia (wie Anm. 10), S. 37.

102 Vgl. Harbig, Die aufgezwungene Sprache (wie Anm. 21), S. 94-96. Bezeichnend für die Intention des adligen Verfassungsentwurfs war neben der Ablehnung des Deutschen auch die völlige Ausklammerung des Ukrainischen. Vgl. Jan Fellerer: Mehrsprachigkeit im galizischen Verwaltungswesen (1772-1914). Eine historisch-soziolinguistische Studie zum Polnischen und Ruthenischen. Köln 2005, S. $86 \mathrm{f}$.

103 Für Kortum bediente sich die Szlachta mit dem Lateinischen einer Sprache, „die schon vor tausend Jahren begraben, und wenige von den Begriffen auszudrüken fähig ist, die unsere erweiterten Kenntnisse, und die Entstehung einer ganz neuen Ordnung der Dinge, nothwendig gemacht haben“. Ernst Traugott von Kortum: Magna Charta von Galizien oder Untersuchung der Beschwerden des galizischen Adels pohlnischer Nation über die österreichische Regierung. Jassy 1790, S. 154.

104 Zit. nach Harbig, Die aufgezwungene Sprache (wie Anm. 21), S. 100. 
Trotz dieser Dramatisierungen musste die repressive Sprachpolitik an den Landschulen in den Folgejahren fallengelassen werden, während der Deutschunterricht an den städtischen Schulen wie auch die sprachliche Situation an den Gymnasien jedoch zementiert wurden. Die Bestrebungen, Galizien durch ,Germanisierung' in die Monarchie zu integrieren, wurden noch bis Mitte der 1840er Jahre fortgeführt und von den galizischen Ständen wiederholt kritisiert. ${ }^{105}$ Aufgrund der negativen Konnotation des Deutschen bei den polnischen Eliten und deren ungebrochenen gesellschaftlichen Einflusses war die dominierende Stellung der deutschen Sprache jedoch nur von begrenzter Dauer und eng an die einseitigen Machtverhältnisse des Vormärz gebunden.

\section{Resümee}

Bis zum Tod Maria Theresias spielten sprachpolitische Fragen primär auf einer konzeptionellen Ebene eine Rolle, während konkrete schulpolitische Maßnahmen aufgrund des schleppenden Verwaltungsaufbaus in Galizien noch rar blieben. Dennoch erfolgten in den 1770er Jahren bereits die entscheidenden Weichenstellungen für die genuin josephinische Politik ab 1780, wurde das Ziel eines öffentlichen Sprachwechsels zugunsten des als Kultursprache verstandenen Deutschen doch bereits in den Diskussionen um die Einführung der Allgemeinen Schulordnung explizit formuliert. Mit dem Wunsch einer Verbreitung der deutschen Sprache, die in theresianischer Zeit noch primär auf den galizischen Adel abzielte, verband sich analog eine aus dem aufklärerischen Polendiskurs sowie dem zeitgenössischen Sprachdiskurs gleichermaßen ererbte Abwertung des Polnischen, das als nicht normierte Umgangssprache auf den Bereich mündlicher Kommunikation beschränkt bleiben sollte. Die Expansion des Deutschen als ,Sprache der Macht‘ im öffentlichen Leben Galiziens wurde bereits vor 1780 kulturmissionarisch legitimiert, wobei die vorgebrachten Argumente die naheliegendere Intention einer staatlichen Herrschaftssicherung in der ,revindizierten' Provinz marginalisierten.

Mit dem Herrschaftsantritt Josephs II. setzte schließlich eine verstärkte Expansion des Deutschen als Staatssprache der in sich vielgestaltigen Habsburgermonarchie ein. Der bereits unter Maria Theresia zu beobachtende Rückbau des Sekundarschulbereichs kulminierte in der Reduktion und Verstaatlichung der galizischen Gymnasien bei gleichzeitig forciertem Ausbau der Volksschulen, wobei die aufklärerischen Maßnahmen zur Verbreitung des Deutschen kulturmissionarisch wie utilitaristisch begründet erscheinen. Ihren pointierten Ausdruck fanden die josephinischen Bestrebungen einer ,Zivilisierung durch Sprache“ im Bereich des jüdischen Normalschulwesens, verband sich doch das Ziel eines Sprachwechsels der galizischen Juden

105 Vgl. ebd., S. 118-127. 
explizit mit dem Wunsch nach deren sittlich-moralischer ,Besserung ${ }^{106}$ Hauptsächlich aufgrund finanzieller wie personeller Überforderung der Schulverwaltung konnten die angestrebten Ziele jedoch nie erreicht werden. Zudem zog die repressive Sprachpolitik neben einem Widerstand jüdischerseits auch eine Widerstandshaltung seitens des polnischen Adels sowie des im Bereich der Lehrkräfte stark vertretenen Klerus nach sich. Vor diesem Hintergrund scheint der missionarische Impetus der höheren Beamtenschaft ab Mitte der 1780er Jahre einer Ernüchterung gewichen zu sein, die bereits die repressiven Maximen der späteren Politik von Kaiser Franz I. im 19. Jahrhundert einleitete. 